\title{
Open-pFind Verified Four Missing Proteins from Multi-tissues
}

Shujia $\mathrm{Wu}^{1,2 \#, ~ J i n s h u a i ~ S u n, 3 \#, ~ X i ~ W a n g ~}{ }^{4}$, Feng $\mathrm{Xu}^{2}$, Hao Chi ${ }^{4}$, Yanchang $\mathrm{Li}^{2}$, Bowen Zhong ${ }^{2}$, Yuping $\mathrm{Xie}^{2}$, Zhonghua Yan², Lei Chang' ${ }^{2}$, Dongxue Wang' ${ }^{2}$, Fuchu $\mathrm{He}^{2 *}$, Junzhu $\mathrm{Wu}^{1 *}$, Yao Zhang ${ }^{2 *}$, Ping $\mathrm{Xu}^{1,2,3^{*}}$

1School of Basic Medical Science, Key Laboratory of Combinational Biosynthesis and Drug Discovery of Ministry of Education, School of Basic Medical Sciences, Wuhan University, Wuhan 430072, China.

${ }^{2}$ State Key Laboratory of Proteomics, Beijing Proteome Research Center, National Center for Protein Sciences (Beijing), Research Unit of Proteomics \& Research and Development of New Drug of Chinese Academy of Medical Sciences, Institute of Lifeomics, Beijing 102206, China.

${ }^{3}$ Hebei Province Key Lab of Research and Application on Microbial Diversity, College of Life Sciences, Hebei University, Baoding, Hebei 071002, China.

${ }^{4}$ Key Laboratory of Intelligent Information Processing of Chinese Academy of Sciences (CAS), Institute of Computing Technology, Beijing 100080, China.

\section{*Corresponding Authors}

Ping Xu, E-mail: xuping_bprc@126.com.

Yao Zhang, E-mail: zhangyaowsw@163.com.

Junzhu Wu, E-mail: wujunzhu@whu.edu.cn

Fuchu He, E-mail: hefc@bmi.ac.cn

Supporting information files: Figure S1-12\&Table S1-9 


\section{Supplementary methods}

\section{Calculation of FDRs at the PSM, Peptide, and Protein Levels.}

Open-pFind were used to estimate the false discovery rate (FDR) at the peptidespectrum-match (PSM) level, at the distinct peptide sequence level, and at the protein level. Briefly, the workflow of Open-pFind consists of two steps: open search and restricted search. First, the MS data are preprocessed by pParse, in which multiple precursor ions corresponding to each tandem mass spectrum are calibrated and extracted. Then, the MS/MS data are searched against the indexed database via the open search module. Next, a few key parameters, such as highly abundant modifications, enzymatic specificity and the mass deviation distribution of precursor ions, are automatically learned by the reranking procedure. Second, MS/MS data are searched again via the restricted module, which is similar to regularly used restricted engines. However, the protein database is reduced, and highly abundant modifications are specified automatically; both of these processes are based on the information learned in the previous step rather than expert experience. The results obtained from both the open and restricted searches are merged and reranked again. Finally, PSMs, peptides and proteins are individually filtered according to the $1 \%$ thresholds. 


\section{Supplementary figures}

\section{Name Supplementary Figure Legends}

Figure S1 Comparison of spectral resolution of 30 human tissues by MaxQuant..

Figure S2 Deep coverage of proteomcis data. (A) The comparison of identified proteins in this study with the HPA database. (B) The comparison of identified proteins in this study with the HPA database. (C) The comparison of identified proteins from single testis tissue in our previous study with MS Kim, et als dataset.

Figure S3 Identification of proteins and peptides from 20 tissues with open-pFind. (A) The number of identified proteins from 20 tissues. (B) The accumulating curve of the identified proteins from 20 tissues. (C) The number of identified peptides from 20 tissues. (D) The accumulating curve of the identified peptides from 20 tissues.

Figure S4 The distribution of uniquely identified proteins' sequence coverage.

Figure S5 Higher sequence coverage in combined multiple tissues than single testis proteomic datasets.

Figure S6 The characteristics of 103 potential MP candidates. (A) Venn diagram of the identified MP candidates in this study with the 1,899 MPs in neXtProt database (2020-01-17). (B) The distribution of candidate PE2-PE4 proteins in 20 tissues. (C) The cumulative curve, (D) The chromosome localization, (E) The molecular weight (MW) distribution, $(F)$ The subcellular localization, $(\mathrm{G})$ The gene ontology (GO) of the 103 identified MP.

Figure S7 Verification of the identified unique peptide from P0C7T8 with the synthetic peptide. (A) Verification of the identified peptide "ALPLGPGASGLLTGTVTLELR" (mzspec: PXD021391:01284_G01_P013188_B00_N07_R1:scan:53817:ALPLGPG ASGLLTGTVTLELR/2) from P0C7T8 by synthetic peptide. (B) Verification of the identified peptide "SQSLHYQELQEGF" (mzspec: PXD021391:01283_H03_P013187_S00_N24_R1:scan:24966:SQSLHYQ ELQEGF/2) from P0C7T8 by synthetic peptide.

Figure S8 Verification of the identified peptide "DIVLEIDDFR" (mzspec:PXD021391:01350_H03_P013681_S00_N24_R1:scan:30177:DI V[Val->GIn]LEIDDFR/2) from Q8WWZ4 by synthetic peptide. 
Figure S9 Verification of the identified unique peptide from Q8IV35 by synthetic peptide and documented peptide in PeptideAtlas. (A) Verification of the identified peptide "KEPEEERPQILEAPSLFK" (mzspec: PXD021391:01320_E02_P013559_S00_N13_R1:scan:27747:KEPEEERP QILEAPSLFK/3) from Q8IV35 by synthetic peptide. (B) Another complement $\mathrm{MS}_{2}$ spectrum of "LNLIATAGINNK" from Q8IV35 in PeptideAtlas.

Figure S10 Verification of the identified unique peptide from 014921 by PeptideAtlas documented peptides. (A) $\mathrm{MS}_{2}$ spectrum of "IYIQPQSPR"(mzspec: PXD021391:01092_E01_P010747_S00_N05_R1:scan:12073:IYIQPQSP $\mathrm{R} / 2$ ) from 014921 in this study. (B) $\mathrm{MS}_{2}$ spectrum of "IYIQPQSPR" from 014921 in PeptideAtlas. (C) $\mathrm{MS}_{2}$ spectrum of “NIQEPTETCFEEAQK"(mzspec:PXD021391:01092_C03_P010747_S00_ N19_R1:scan:18260:NIQEPTETC[Carbamidomethyl]FEEAQK/2) from O14921 in this study. (D) $\mathrm{MS}_{2}$ spectrum of "NIQEPTETCFEEAQK" from O14921 in PeptideAtlas.

Figure S11 Verification of the identified peptide "LQESGPGLVKPSQTLSLTCTVSGGSISSGD"

(mzspec:PXD021391:01294_G01_P013197_S00_N07_R1:scan:30519:L QESGPGLVKPSQTLSLTCTVSGGSISS[Ser->Val]GD/3) from P0DP06 by synthetic peptide.

Figure S12 Verification of the identified unique peptide from Q8N912 by PeptideAtlas documented peptides. (A) $\mathrm{MS}_{2}$ spectrum of "TAAGAVSPDSRPETR" (mzspec:

PXD021391:01296_G01_P013201_S00_N07_R1:scan:5104:TAAGAVSP DSRPETR/3) from Q8N912 in this study. (B) $\mathrm{MS}_{2}$ spectrum of "TAAGAVSPDSRPETR" from Q8N912 in PeptideAtlas. (C) Another complement $\mathrm{MS}_{2}$ spectrum of "NEEAAWGPR" from Q8N912 in SRMAtlas. (D) $\quad \mathrm{MS}_{2} \quad$ spectrum of "PVATALEDLR"(mzspec:PXD021391:01279_D01_P013163_B00_N04_R1 :scan:13637:P[Pro->Thr]VATALEDLR/3) from Q8N912 in this study. 


\section{Supplementary tables}

\section{Name Supplementary table legends}

Table S1 Searching criteria and results of the proteomics.

Table S2 Detailed information for the identified proteins from 20 tissues.

Table S3 The identified peptides of the 103 MP candidates.

Table S4 Filtration of MP candidates identified after unique peptide length $\geq 9$ AAs.

Table S5 SAAV's filtration of the MP candidates

Table S6 The identification of MP candidates after spectrum manual checking.

Table S7 The survived MP candidates identified after non-nested unique peptides $\geq 2$.

Table S8 Detailed information for four verified MPs according to strict filtering rules.

Table S9 The characterization of the 103 candidates MPs. 


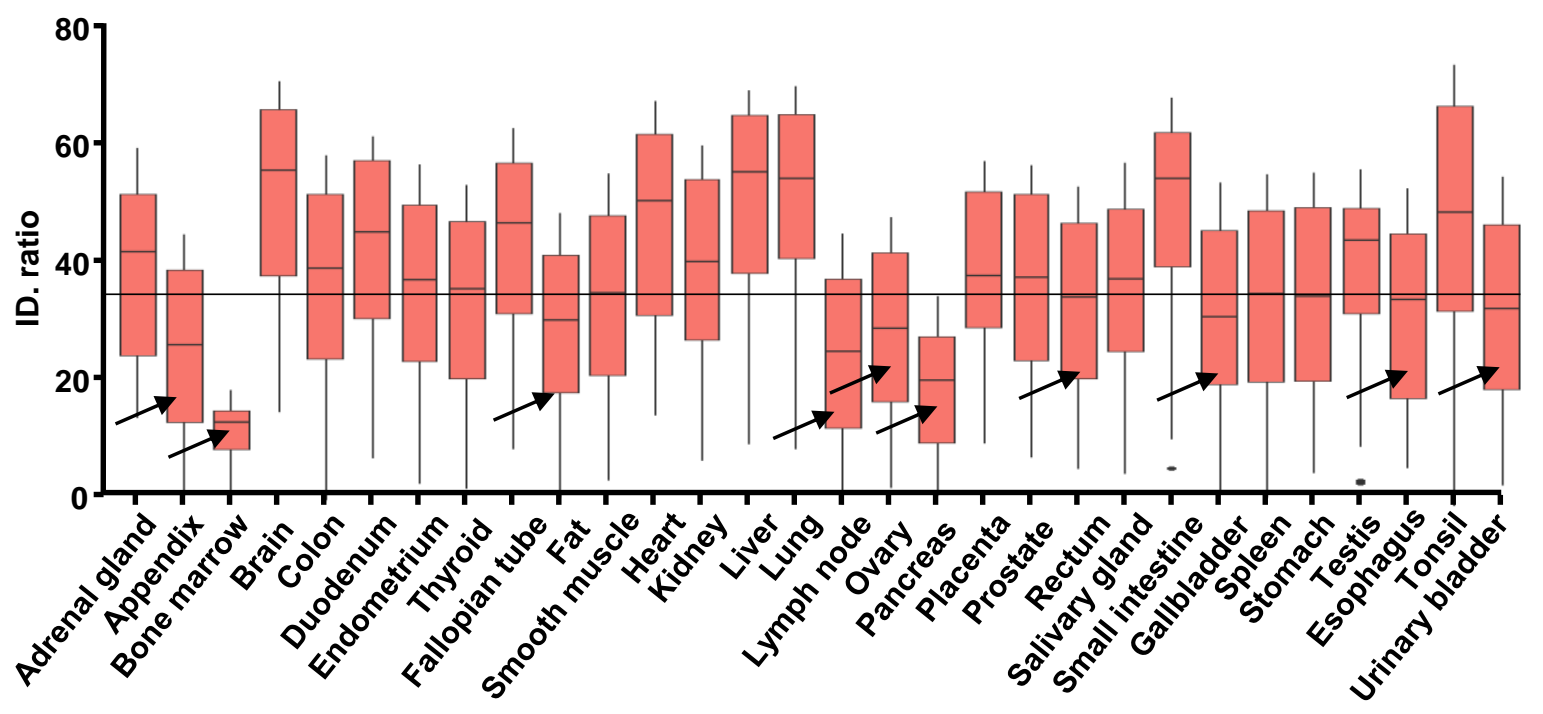

Figure S1. Comparison of spectrum resolution of $\mathbf{3 0}$ human tissues by MaxQuant. 
A

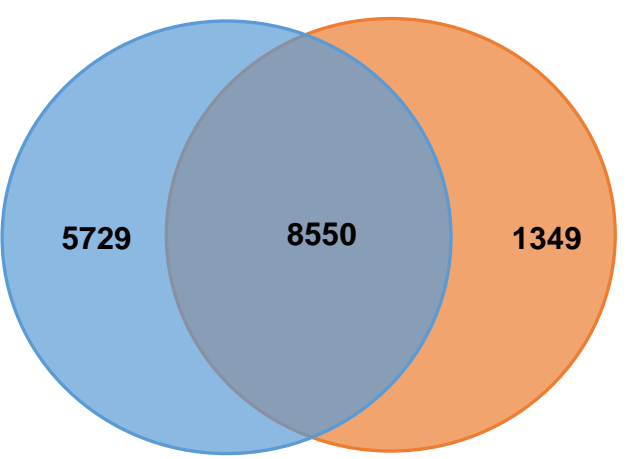

This study
Human Proteome Atlas

Database
C

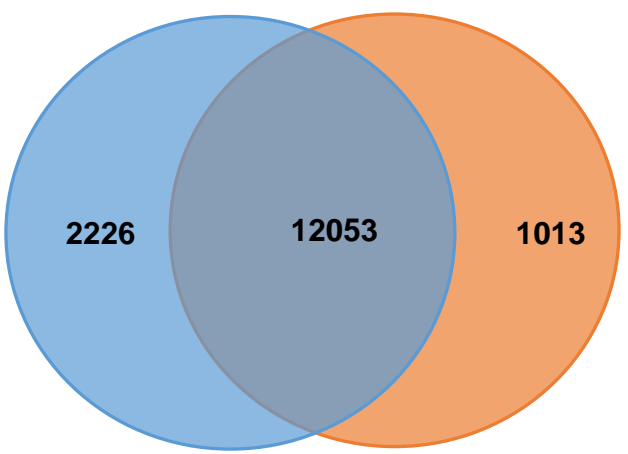

Sun, et al. 2019

B

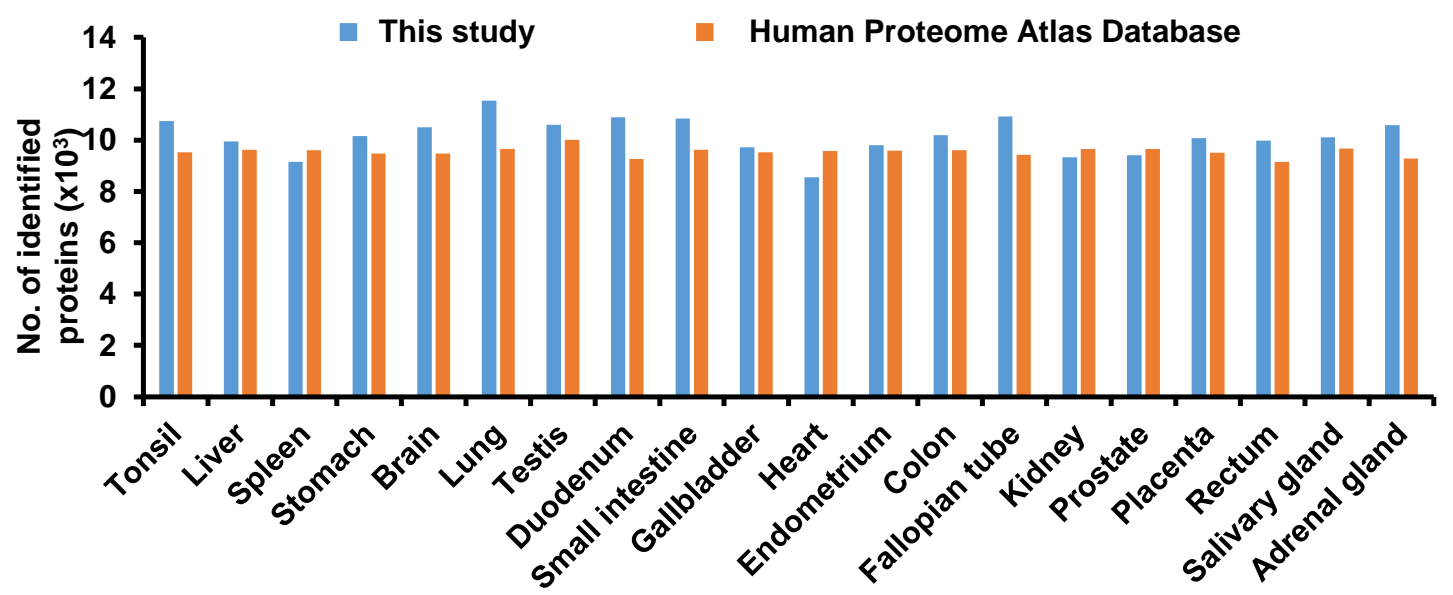

Figure S2. Deep coverage of proteomcis data. (A) The comparison of identified proteins in this study with the HPA database. (B) The comparison of identified proteins in this study with the HPA database. (C) The comparison of identified proteins from single testis tissue in our previous study with MS Kim, et als dataset. 
A

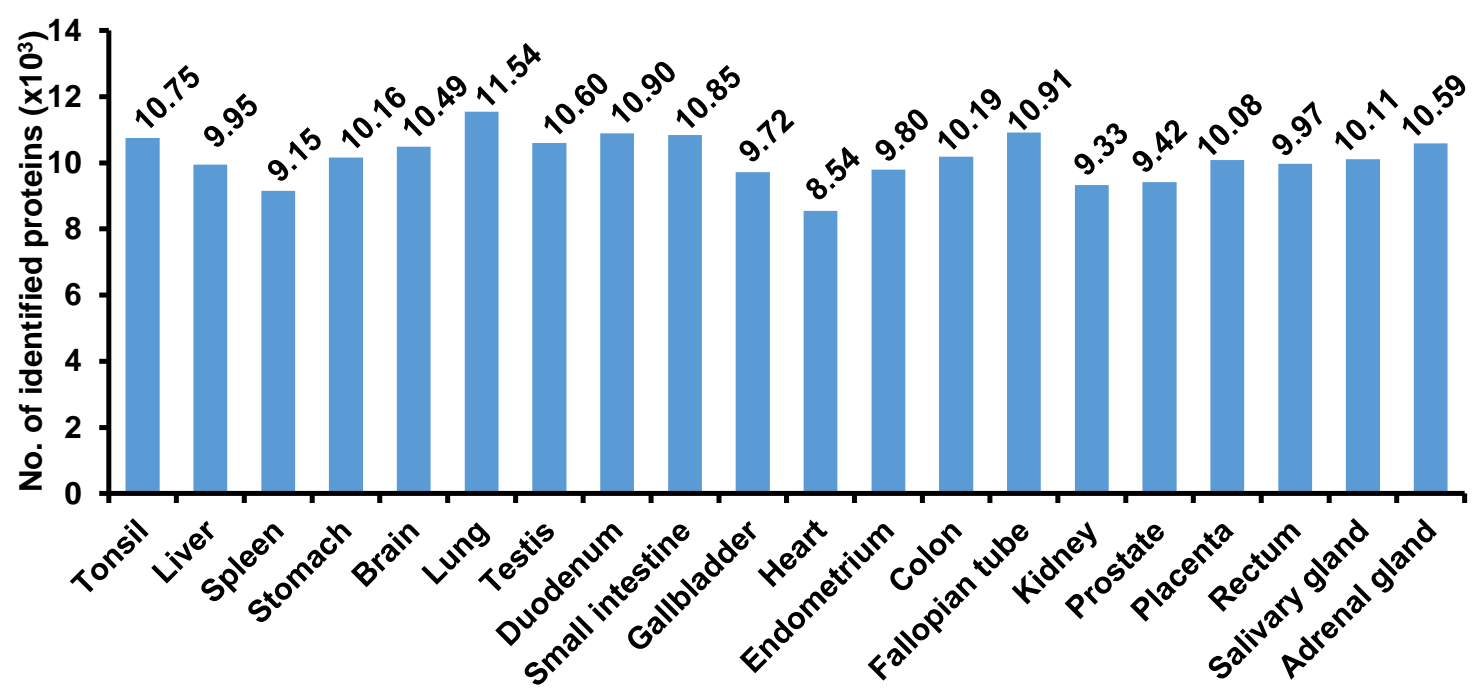

B

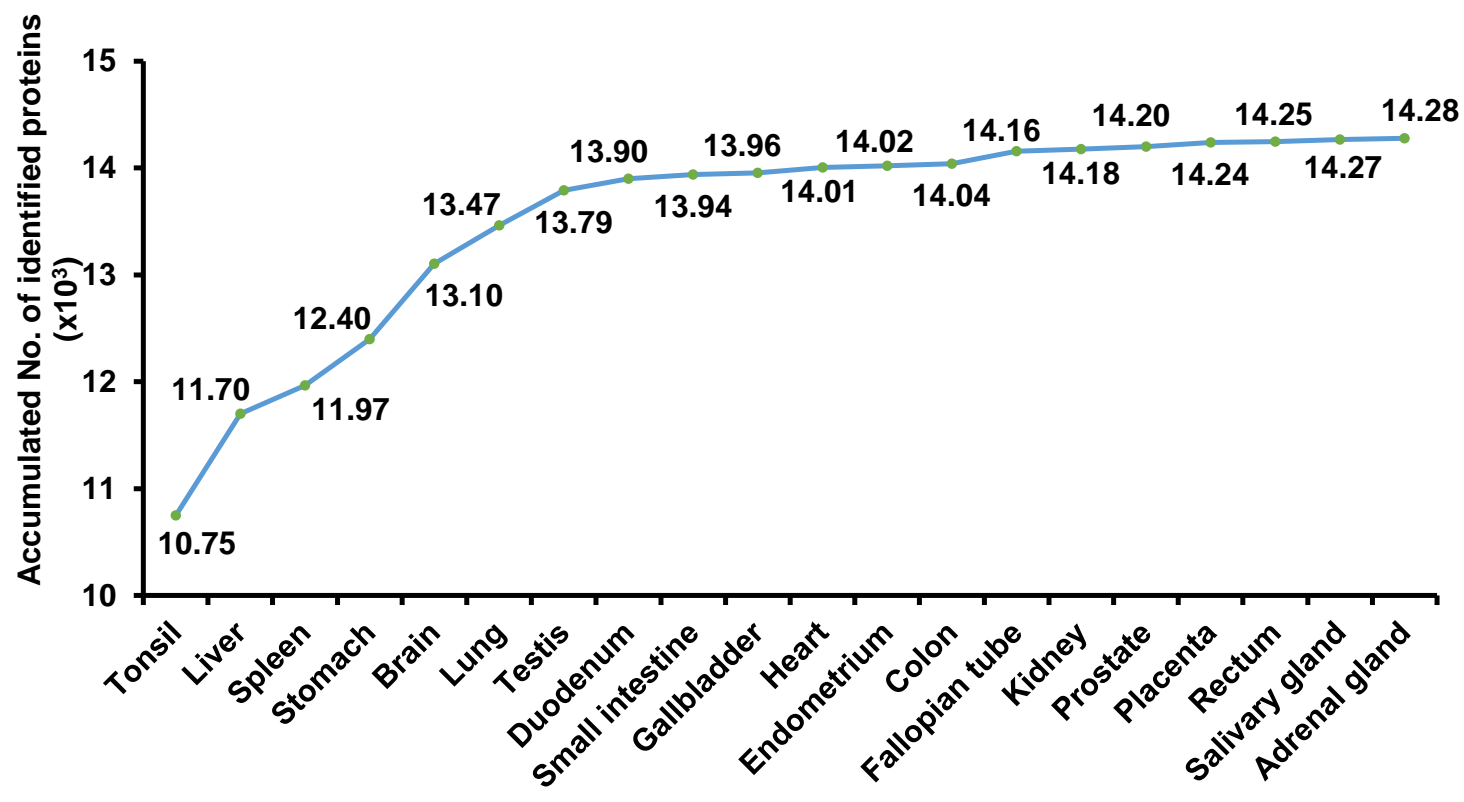


C

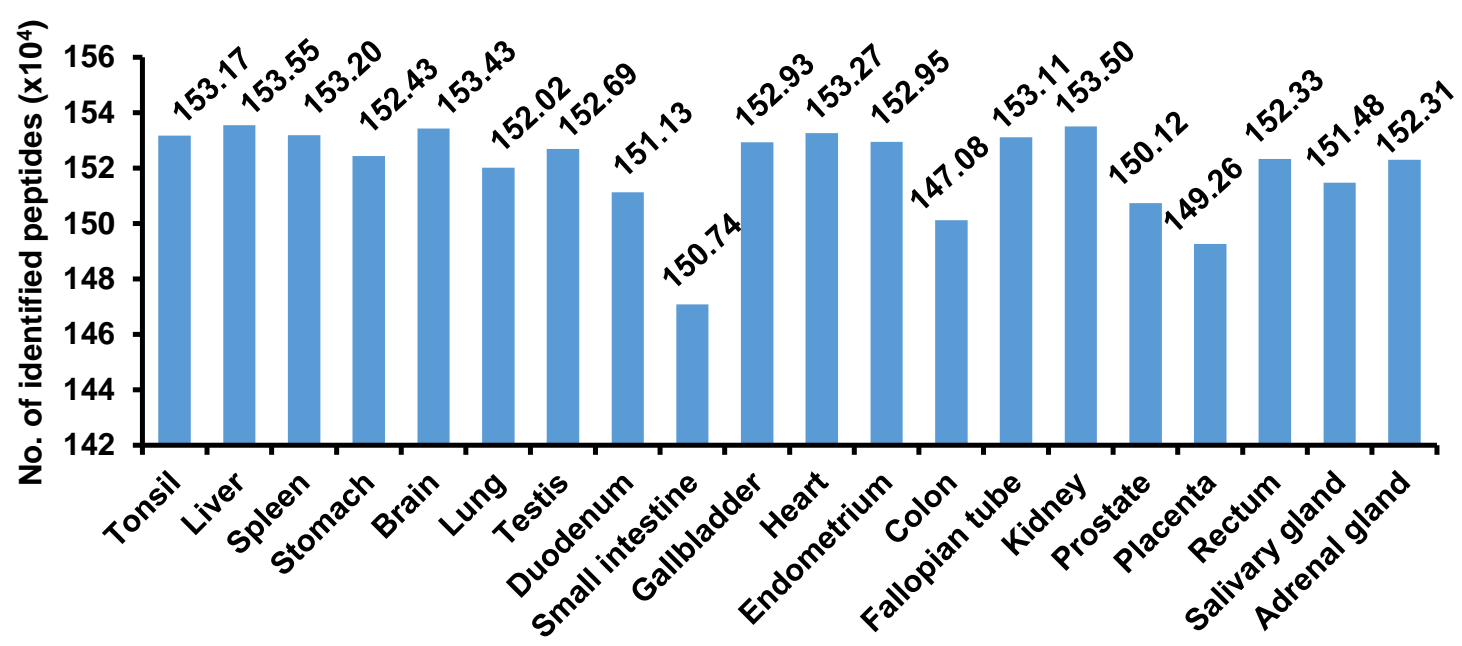

D

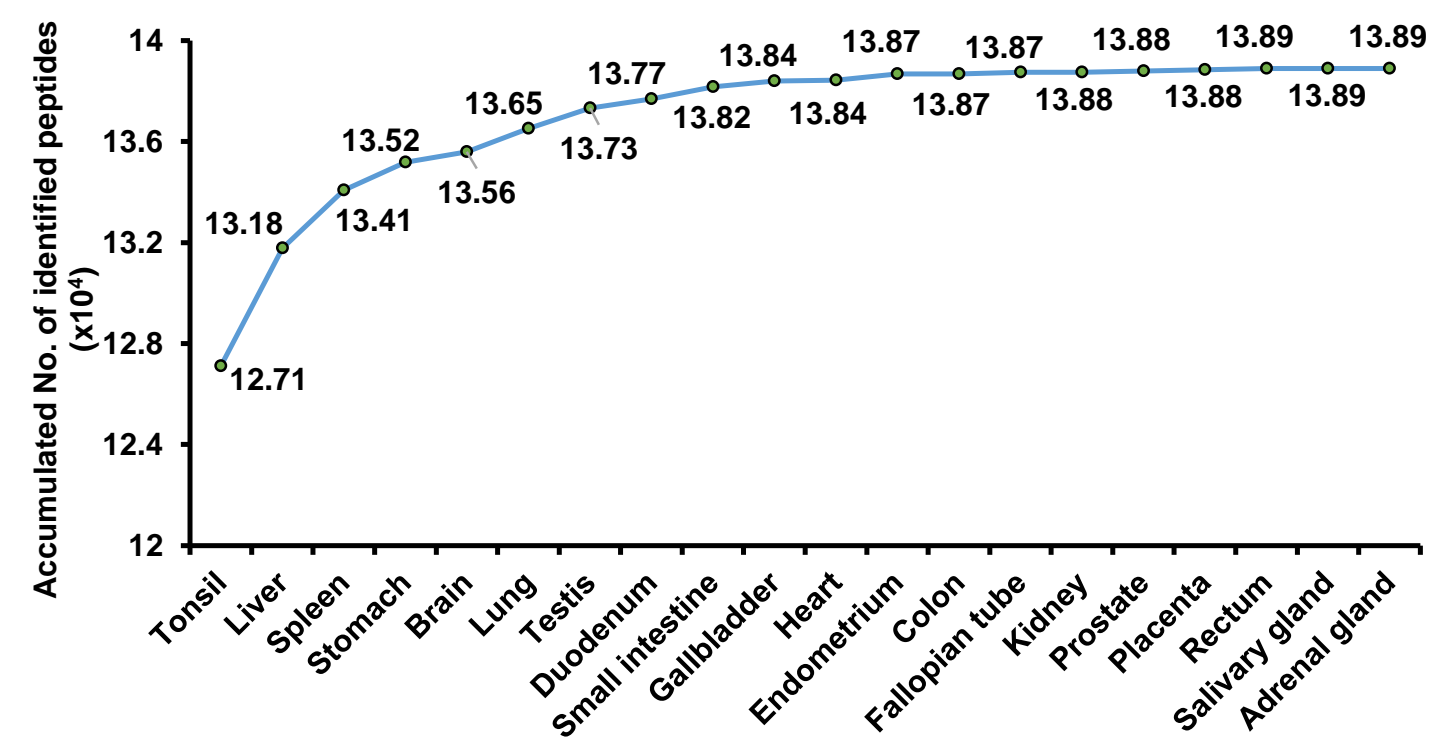

Figure S3. Identification of proteins and peptides from 20 tissues with open-pFind. (A) The number of identified proteins from 20 tissues. (B) The accumulating curve of the identified proteins from 20 tissues. (C) The number of identified peptides from 20 tissues. (D) The accumulating curve of the identified peptides from 20 tissues. 


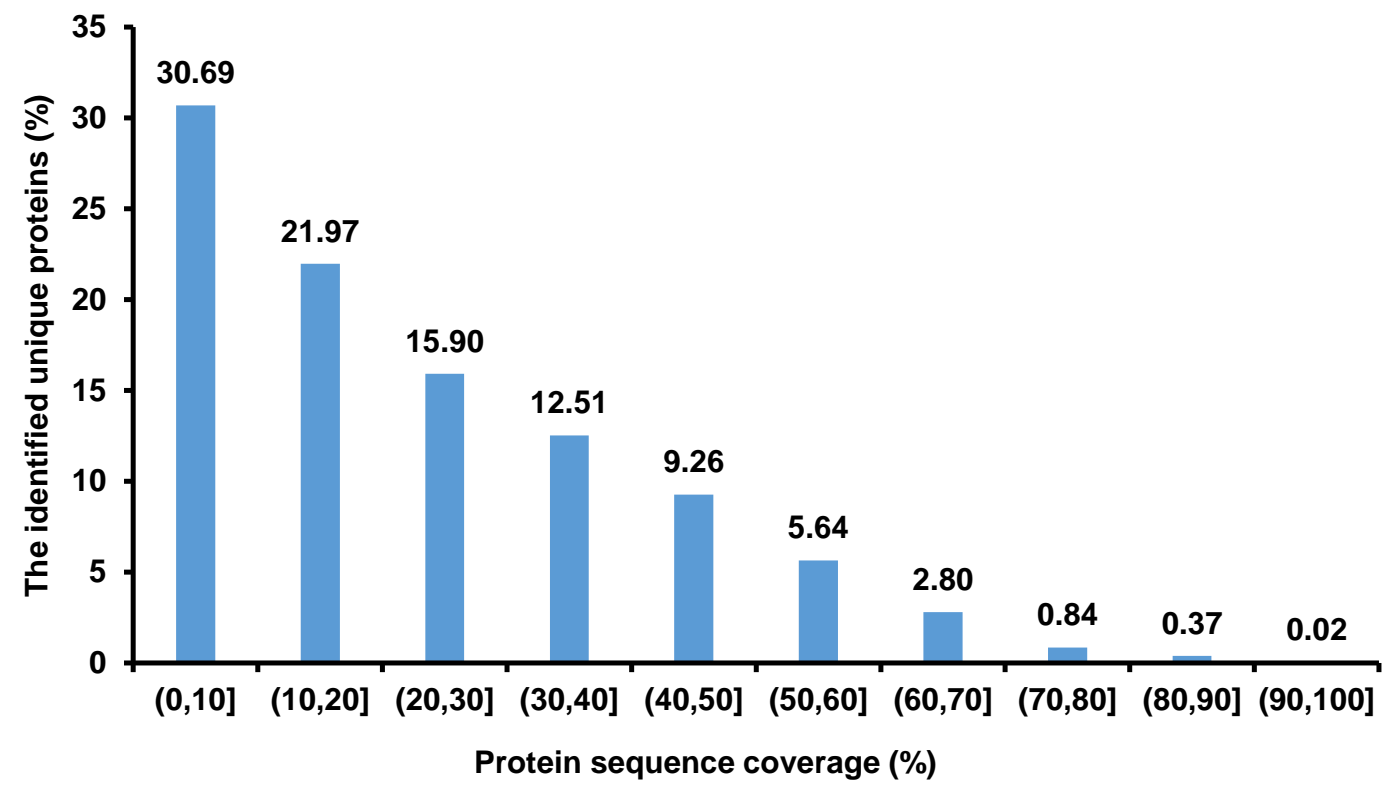

Figure S4. The distribution of uniquely identified proteins' sequence coverage. 


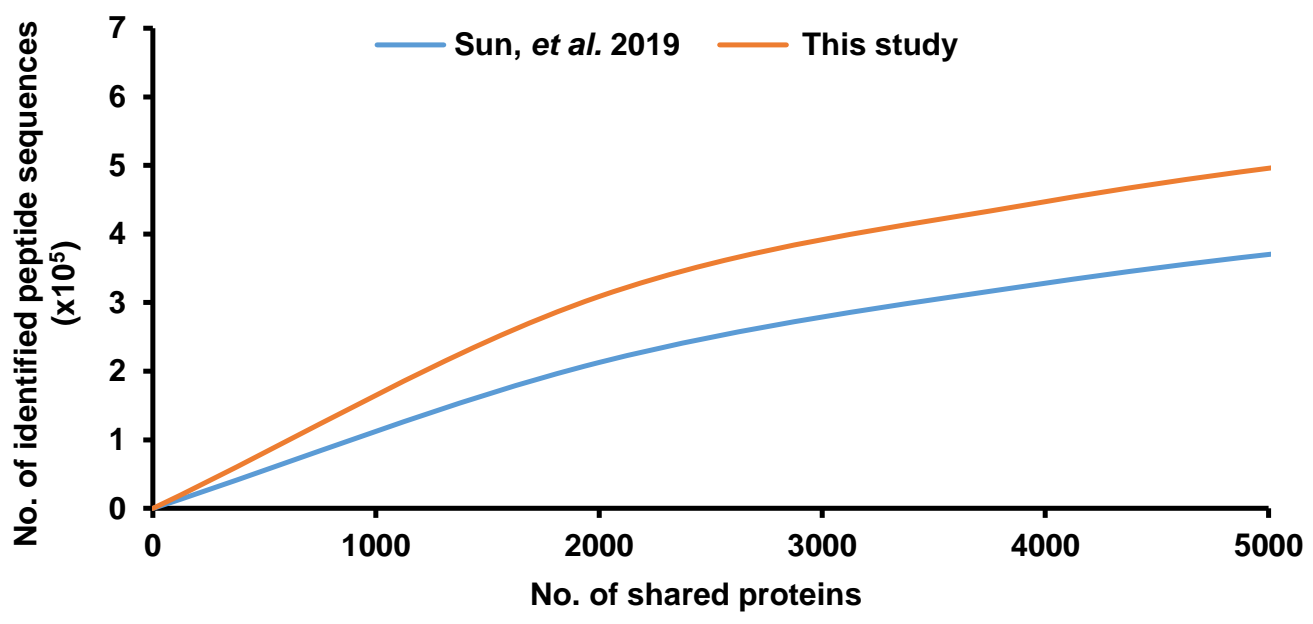

Figure S5. Higher sequence coverage in combined multiple tissues than single testis proteomic datasets. 
A

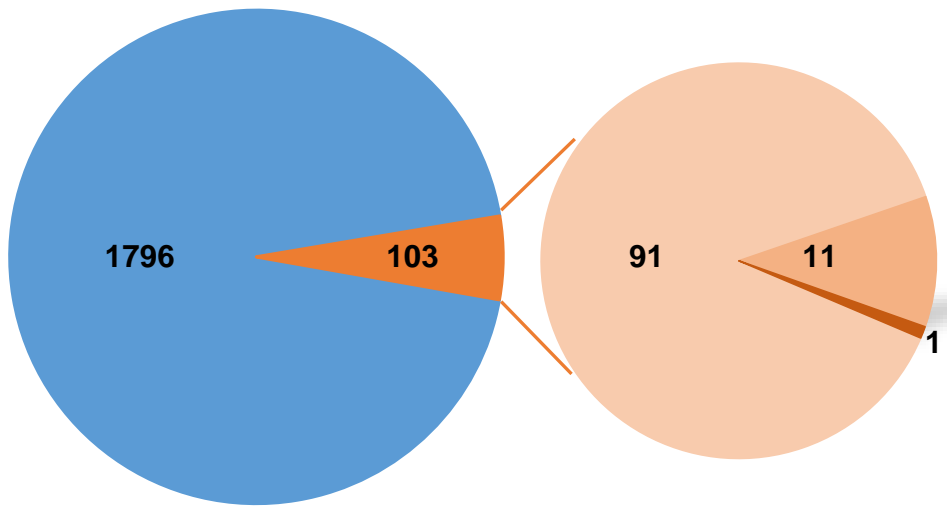

2020 MPs Potential MPs

PE2 PE3 $\square$ PE4

B

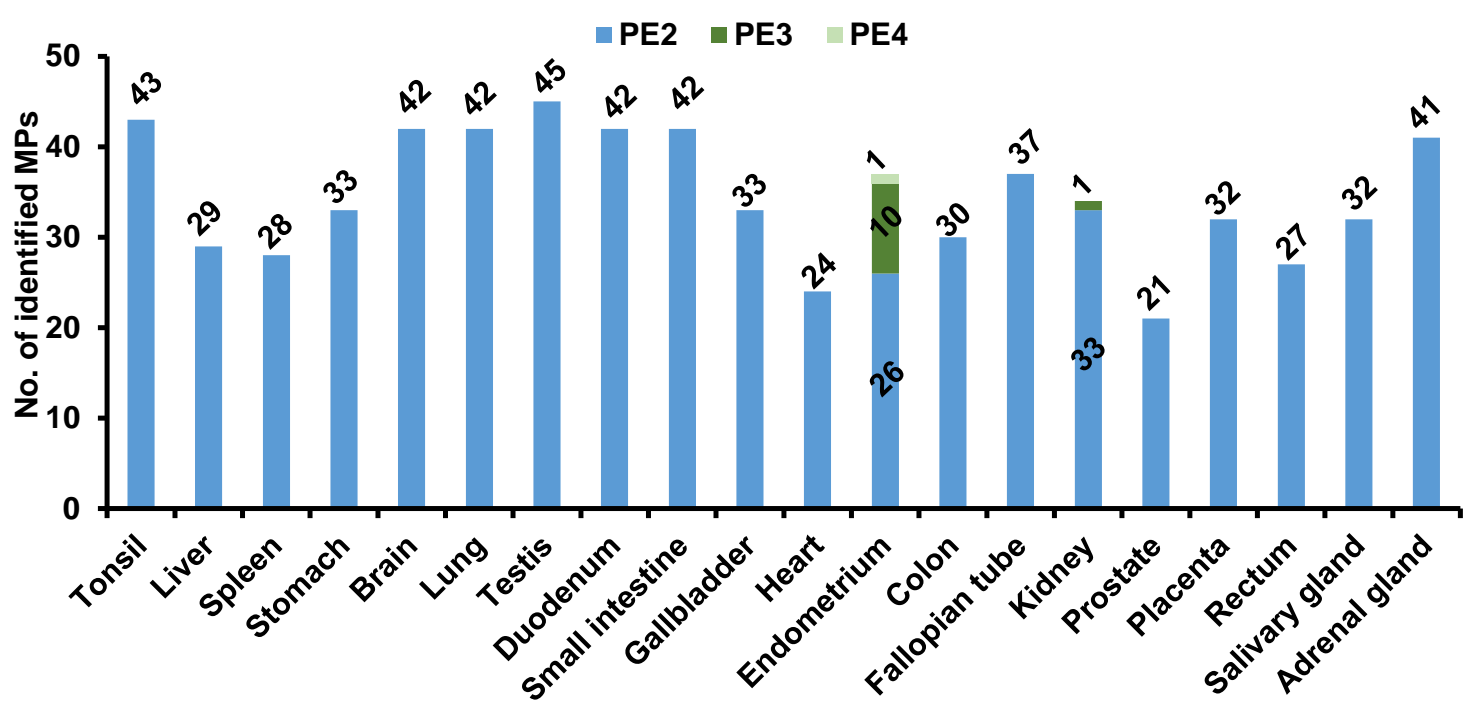

C

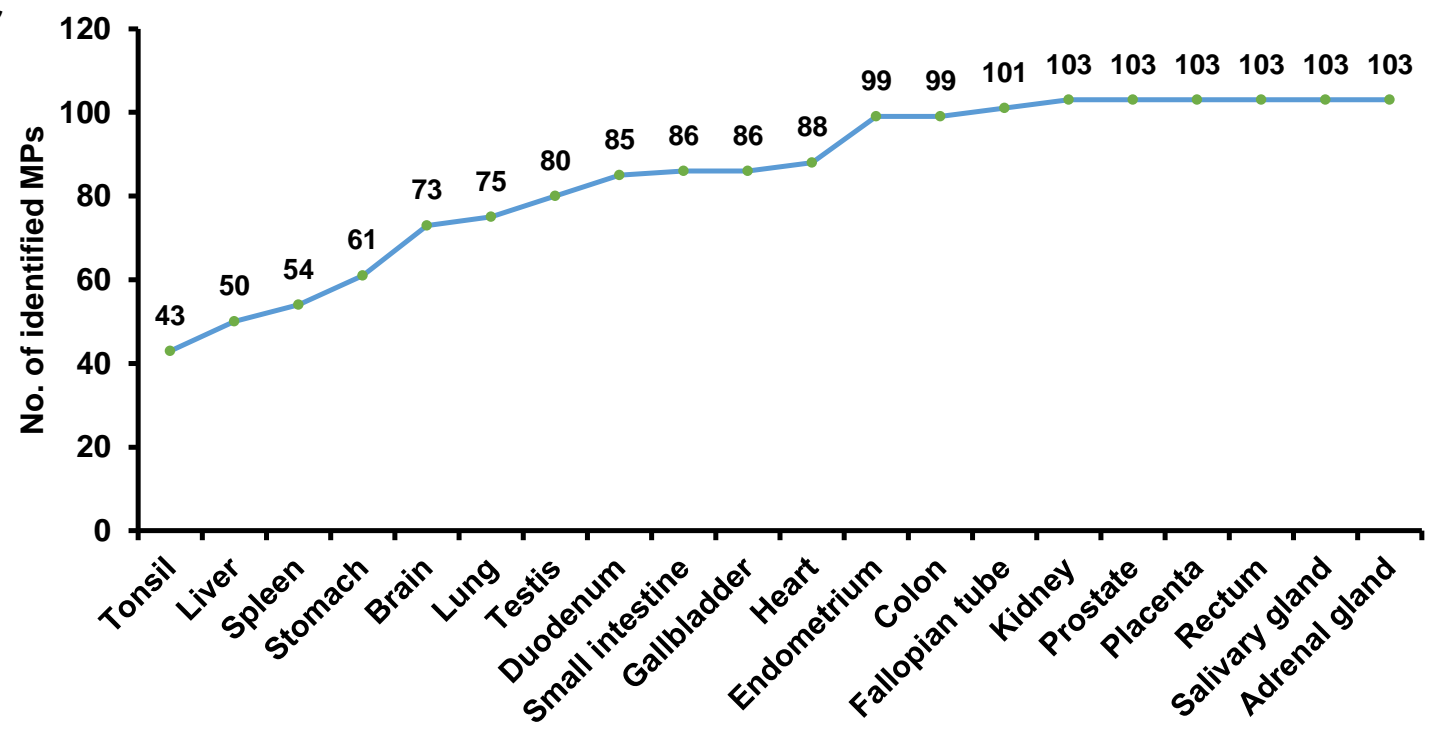


D

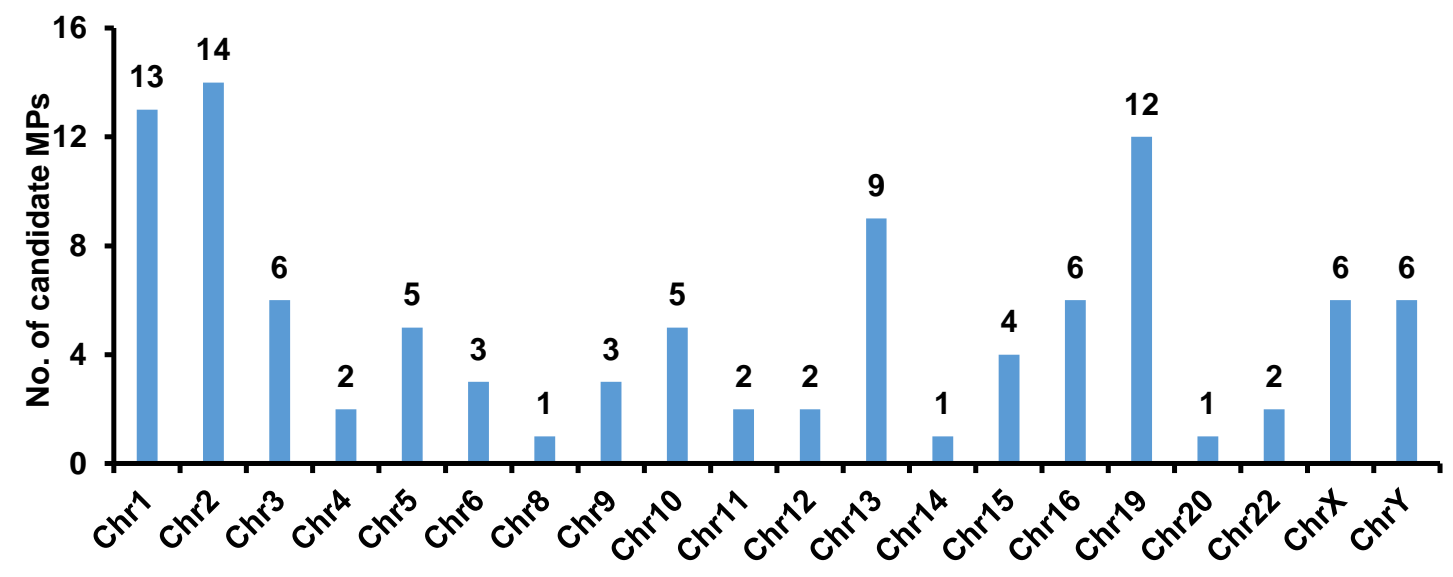

E

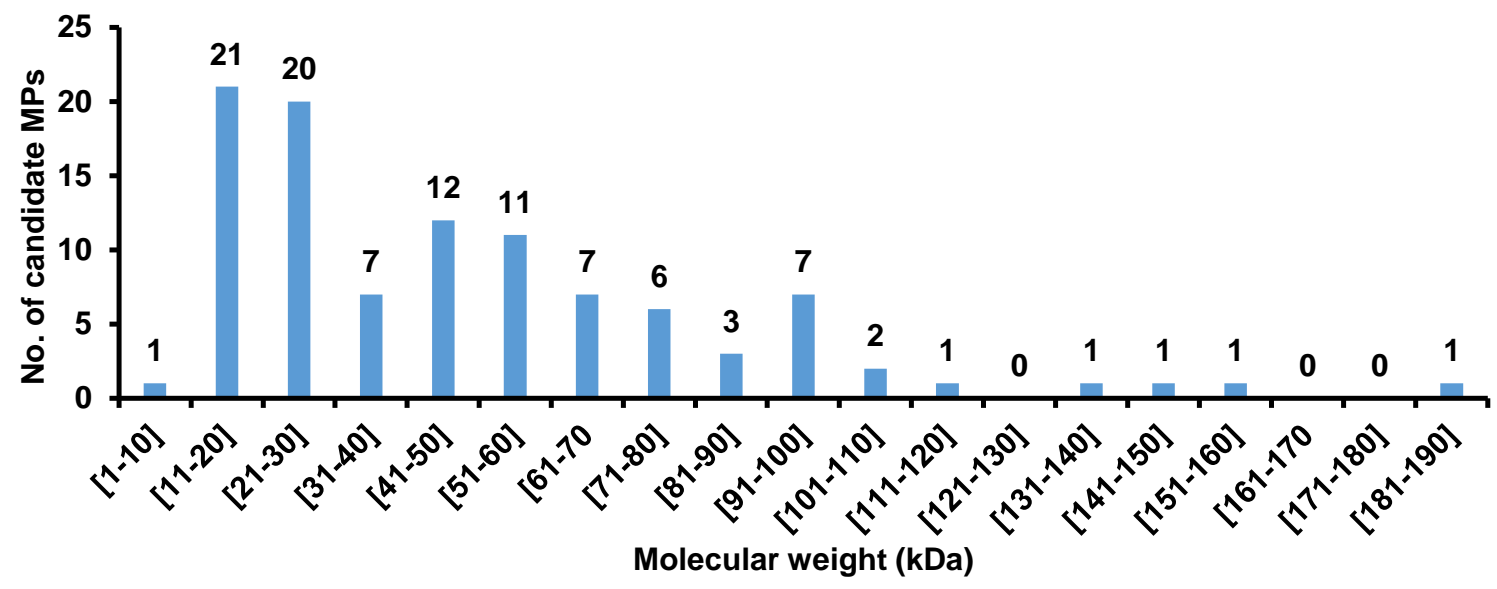

F

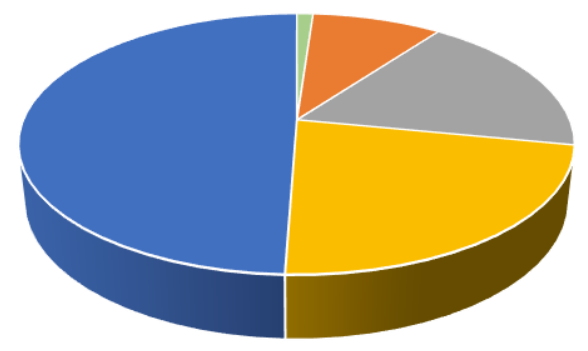

Cell junction

- Nucleus

- Membrane
- Cytoplasm

Secreted 


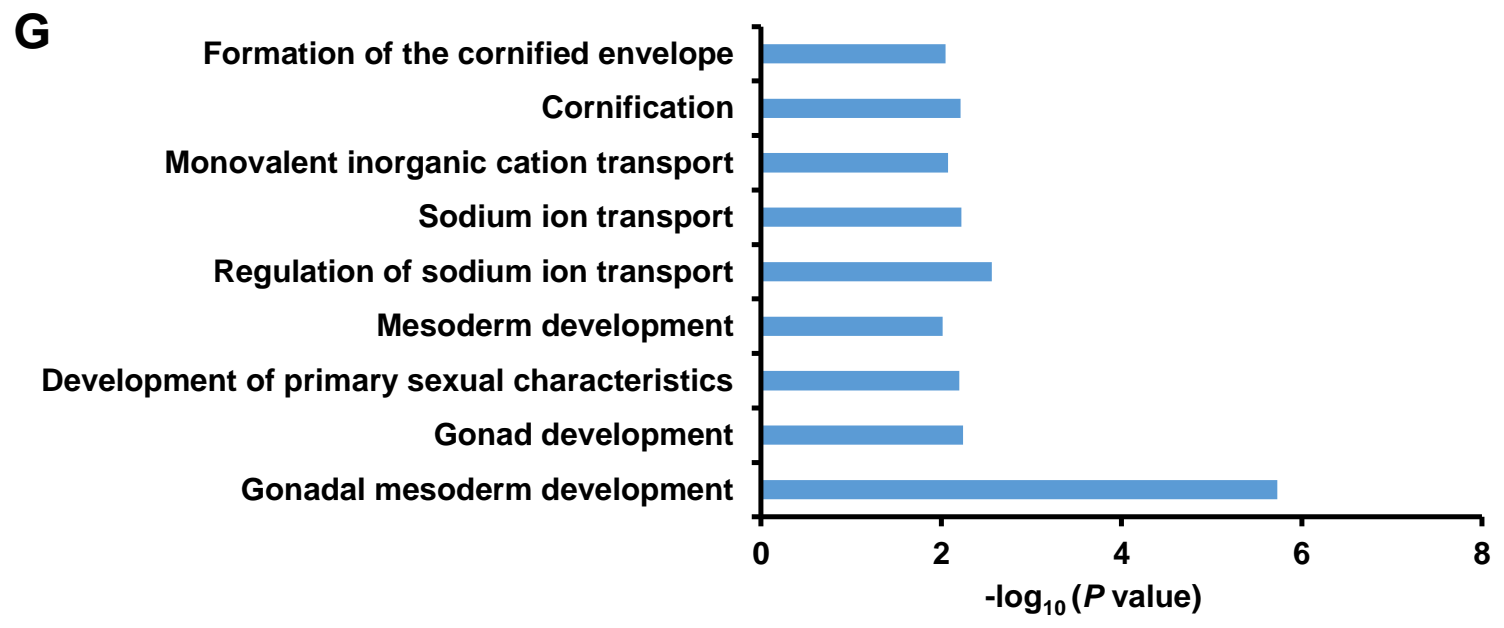

Figure S6. The characteristics of 103 potential MP candidates. (A) Venn diagram of the identified MP candidates in this study with the1,899 MPs in neXtProt database (2020-01-17). (B) The distribution of candidate PE2-PE4 proteins in 20 tissues. (C) The cumulative curve, (D) The chromosome localization, (E) The molecular weight (MW) distribution, $(F)$ The subcellular localization, $(G)$ The gene ontology (GO) of the 103 identified MP. 
A

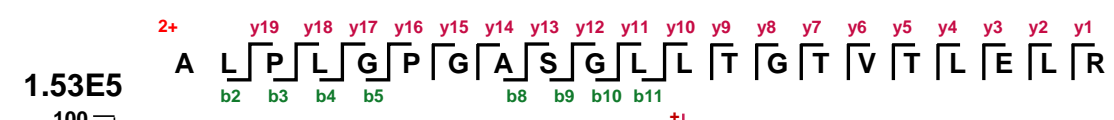

Original
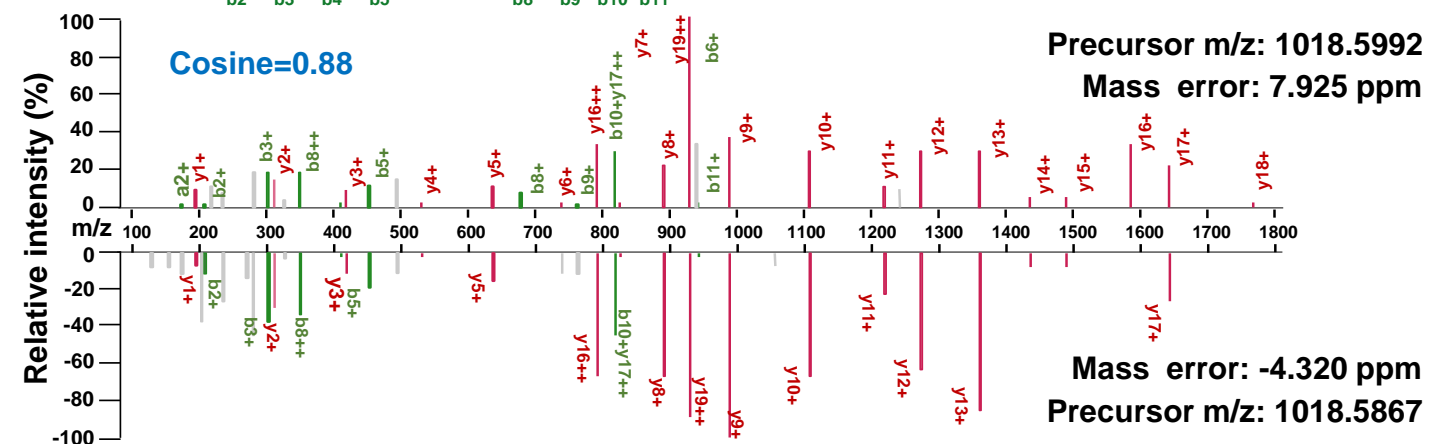

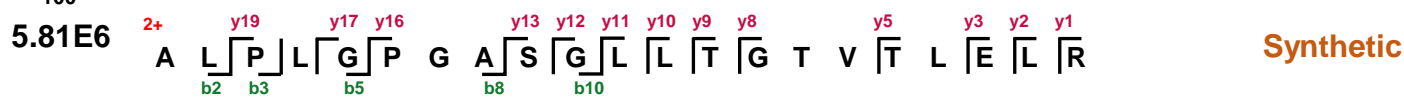

B

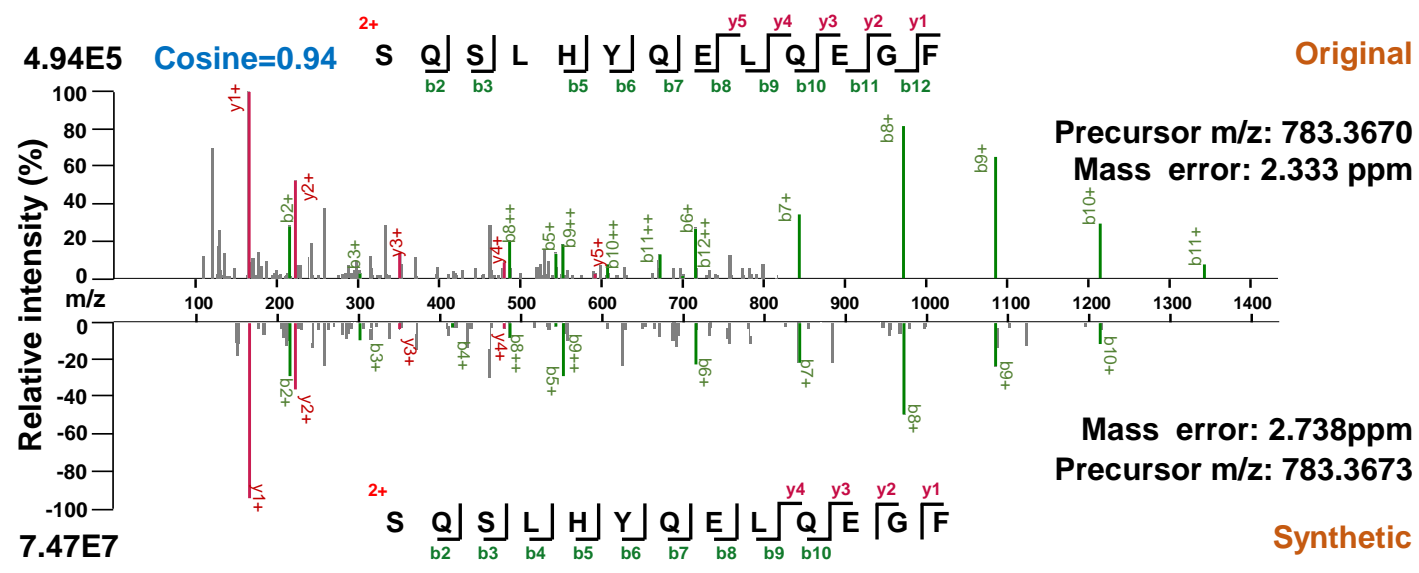

Figure S7. Verification of the identified unique peptide from P0C7T8 with the synthetic peptide. (A) Verification of the identified peptide "ALPLGPGASGLLTGTVTLELR" (mzspec:PXD021391:01284_G01_P013188 _B00_N07_R1:scan:53817:ALPLGPGASGLLTGTVTLELR/2) from P0C7T8 by synthetic peptide. (B) Verification of the identified peptide "SQSLHYQELQEGF"(mzspec:PXD021391:01283_H03_P013187_S00_N24_R1: scan:24966:SQSLHYQELQEGF/2) from P0C7T8 by synthetic peptide. 


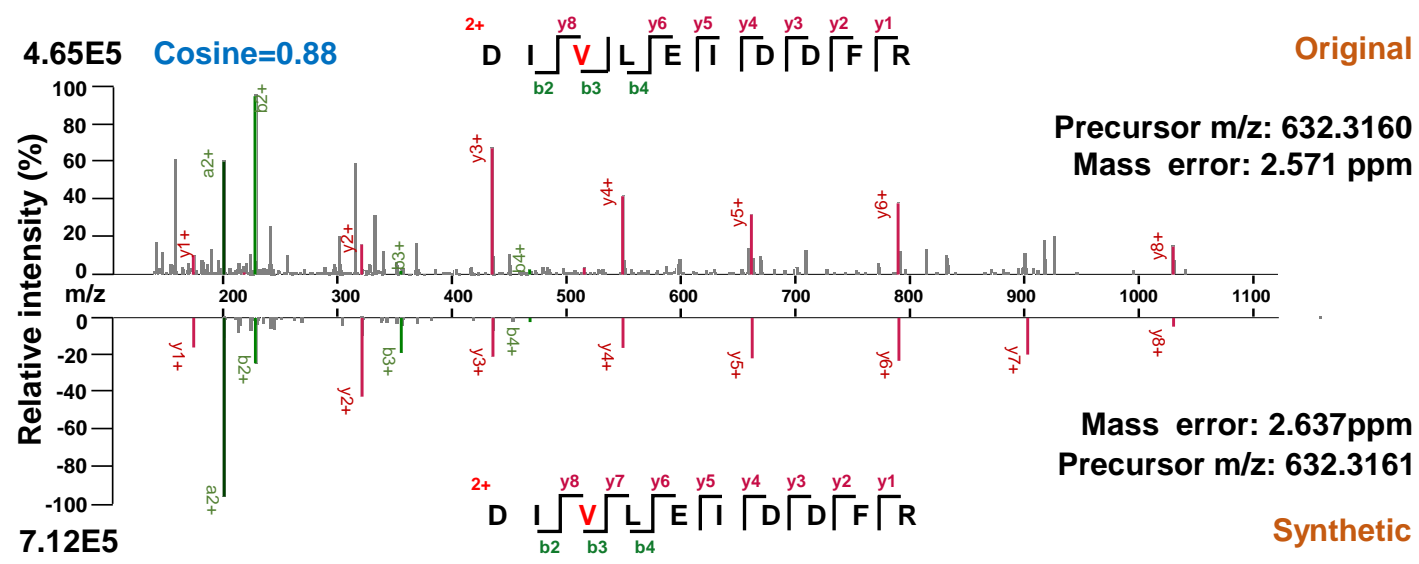

Figure S8. Verification of the identified peptide "DIVLEIDDFR" (mzspec:PXD021391:01350_H03_P013681_S00_N24_R1:scan:30177:DIV[ Val->GIn]LEIDDFR/2) from Q8WWZ4 by synthetic peptide. 
A

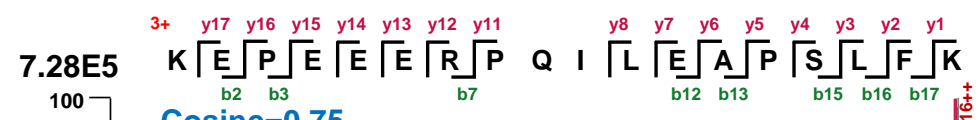

Original

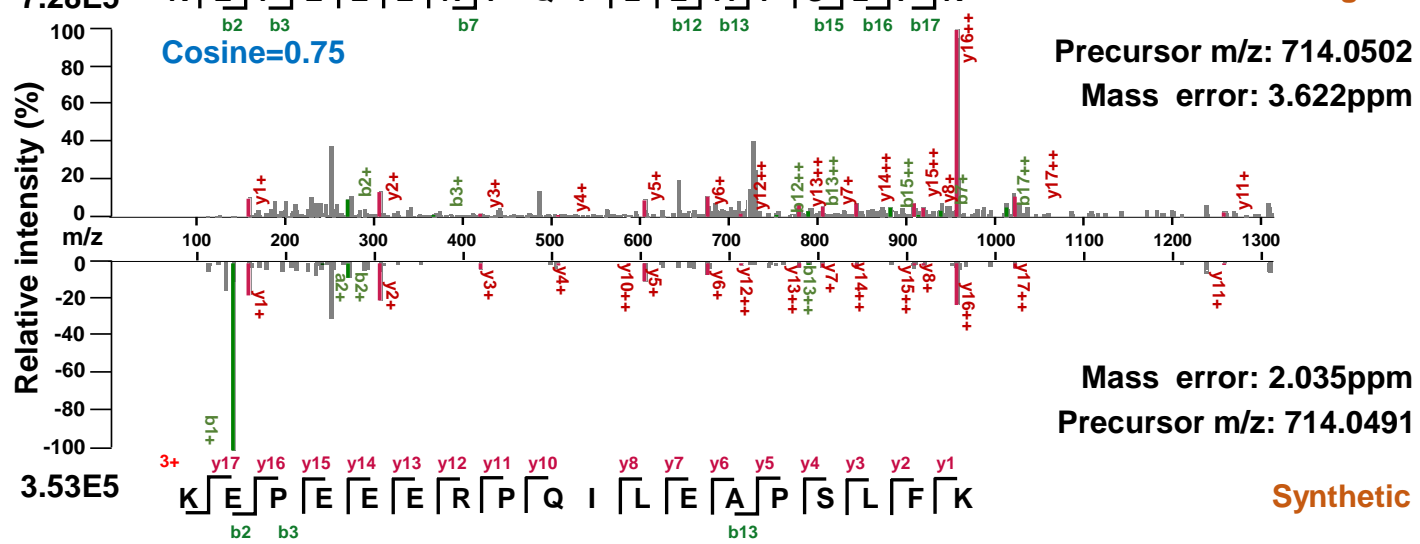

B

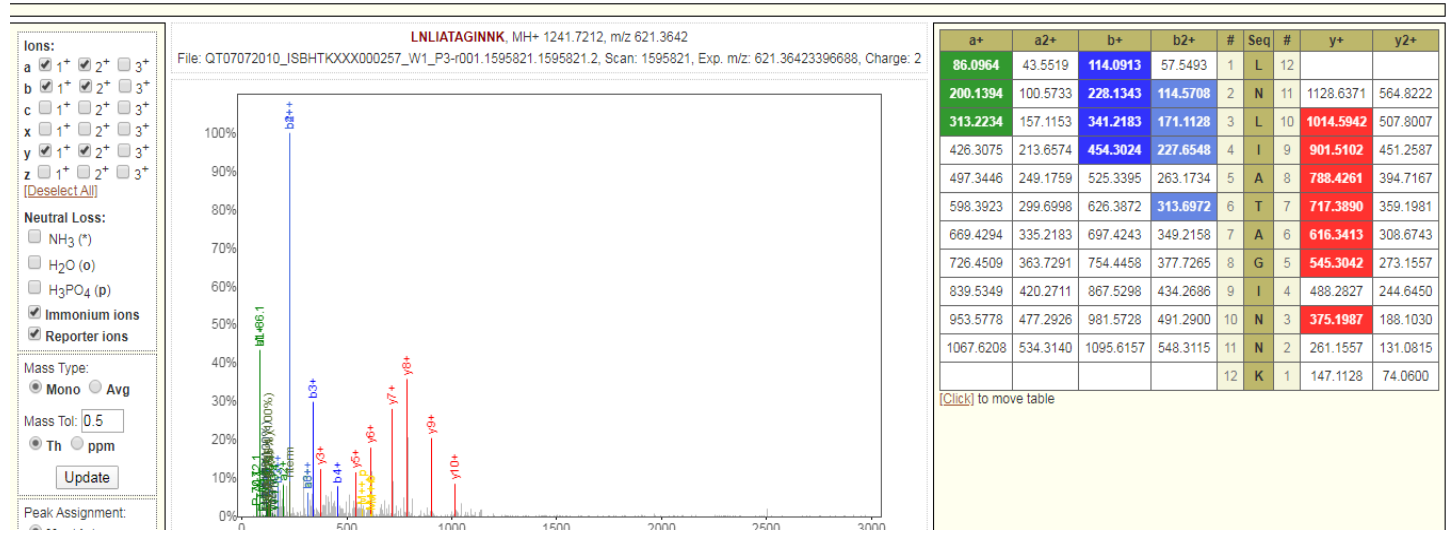

Figure S9. Verification of the identified unique peptide from Q8IV35 by synthetic peptide and documented peptide in PeptideAtlas. (A) Verification of the identified peptide "KEPEEERPQILEAPSLFK" (mzspec: PXD021391:01320_E02_P013559_S00_N13_R1:scan:27747:KEPEEERPQILEAPS LFK/3) from Q8IV35 by synthetic peptide. (B) Another complement $\mathrm{MS}_{2}$ spectrum of "LNLIATAGINNK" from Q8IV35 in PeptideAtlas. 
A

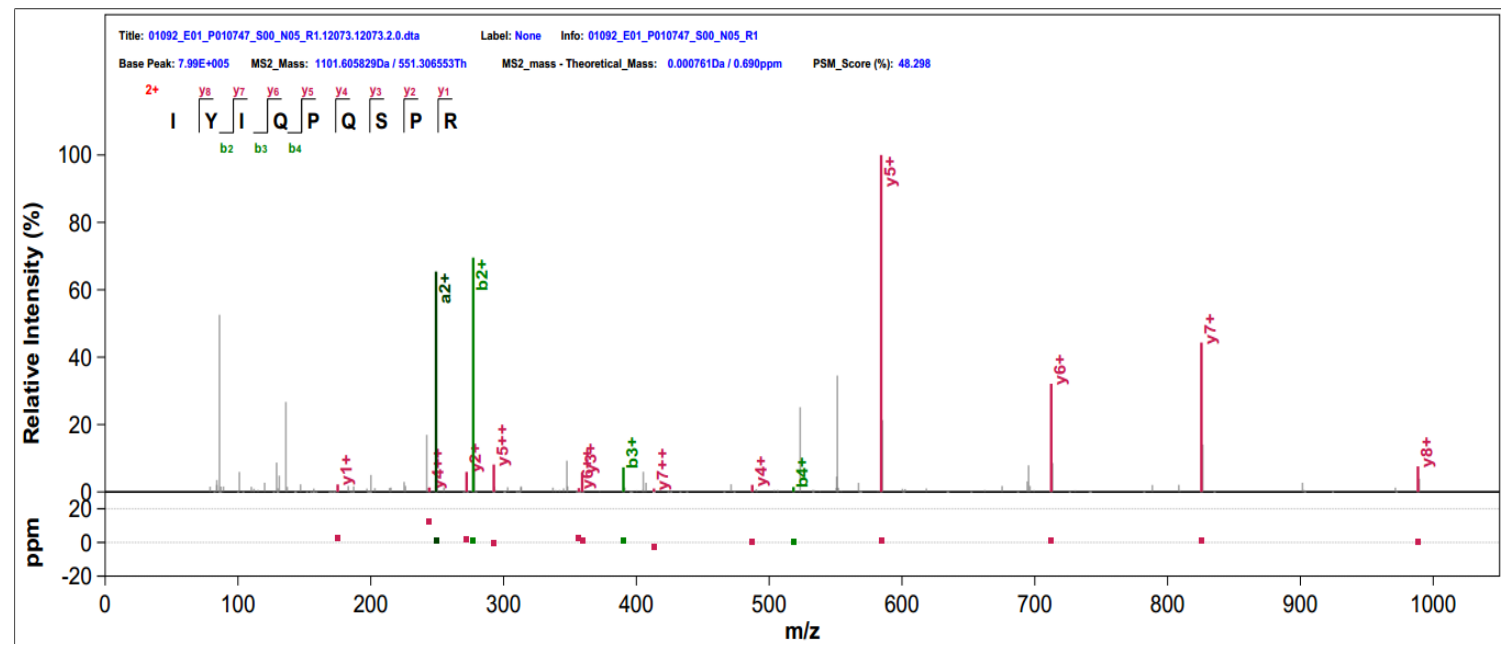

B

Spectrum for IYIQPQSPR +2

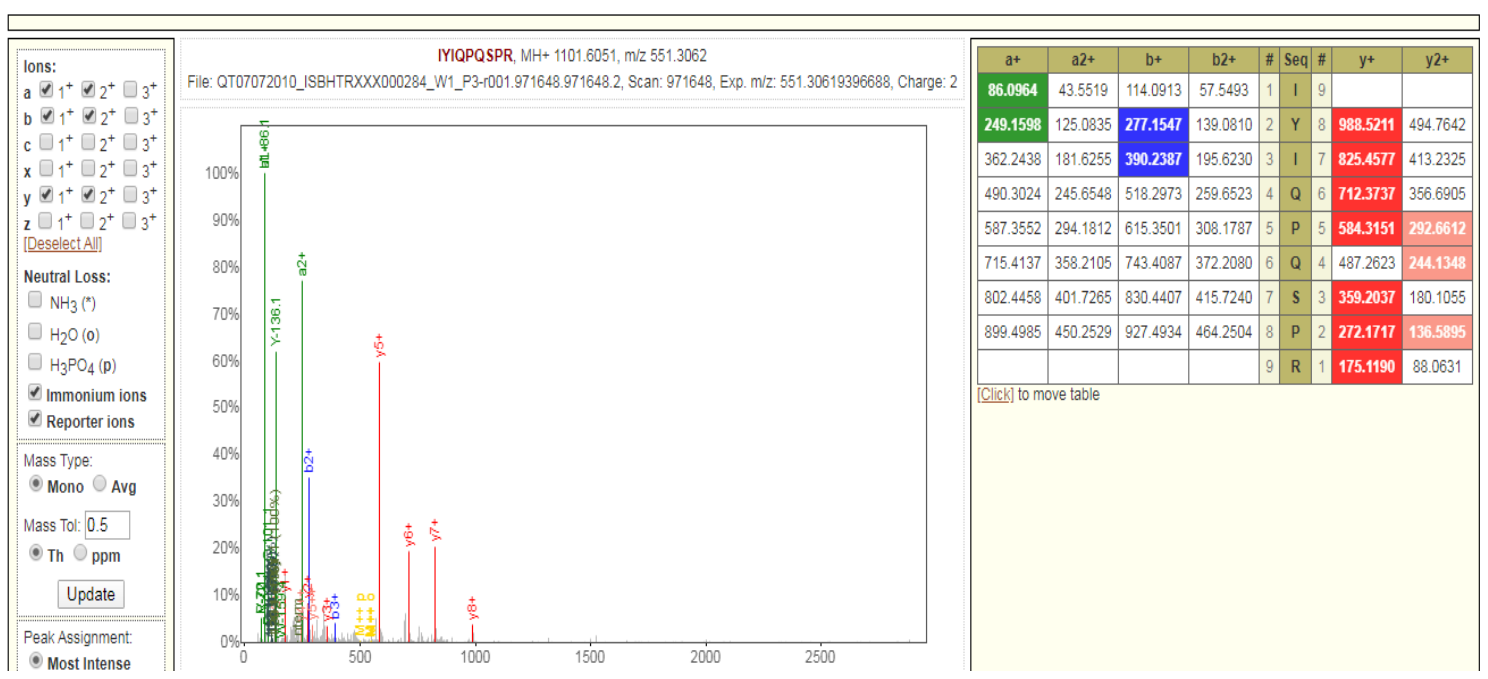


C

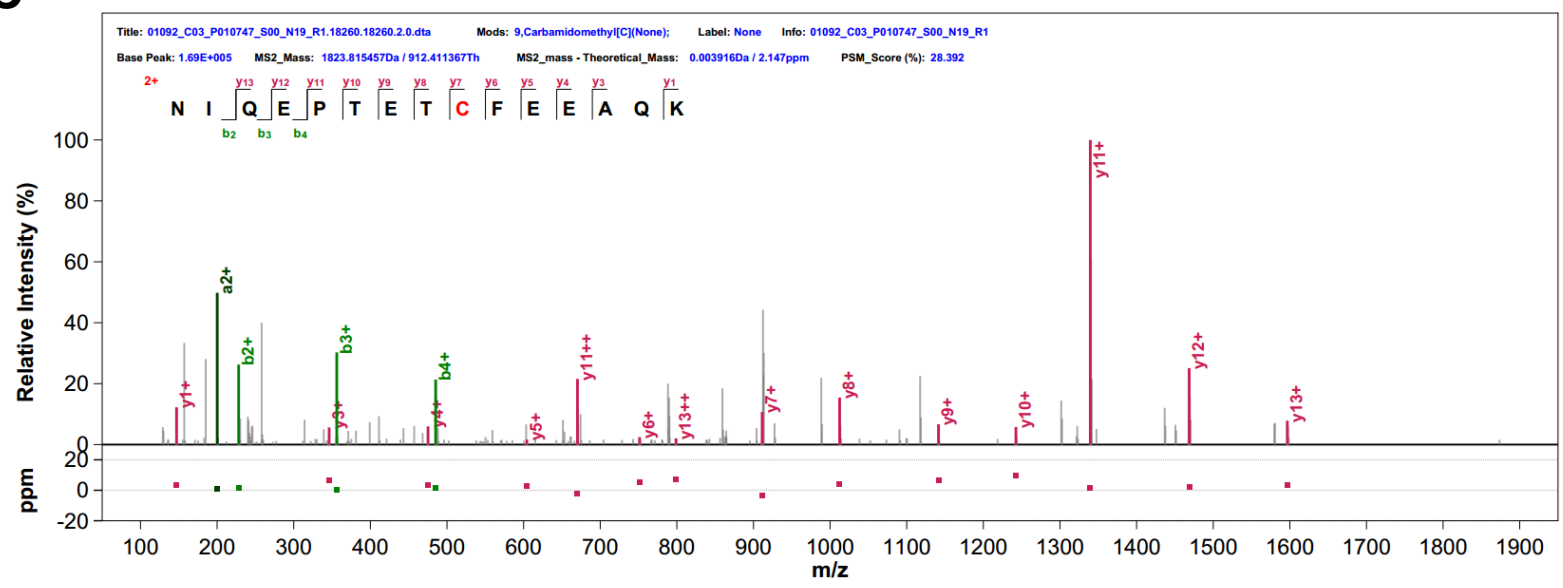

D

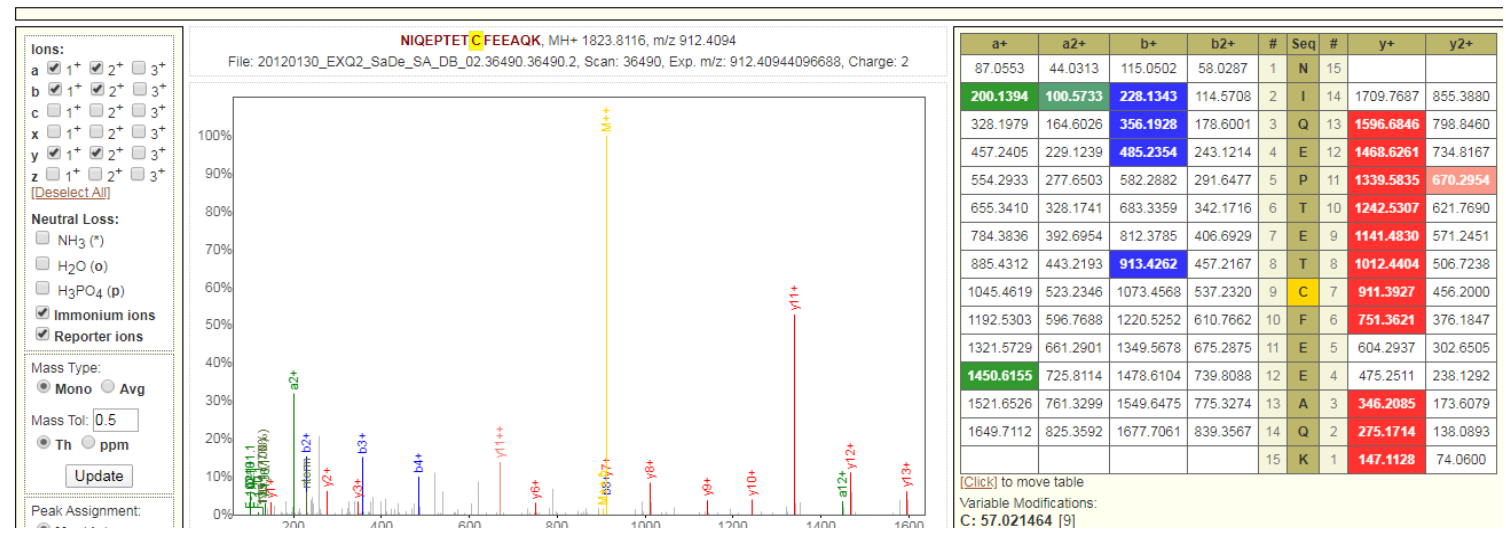

Figure S10. Verification of the identified unique peptide from 014921 by PeptideAtlas documented peptides. (A) $\mathrm{MS}_{2}$ spectrum of "IYIQPQSPR"(mzspec: PXD021391:01092_E01_P010747_S00_N05_R1:scan:12073:IYIQPQSPR/2) from O14921 in this study. (B) $\mathrm{MS}_{2}$ spectrum of "IYIQPQSPR" from 014921 in PeptideAtlas. $\quad$ (C) $\mathrm{MS}_{2}$ spectrum of "NIQEPTETCFEEAQK"(mzspec:PXD021391:01092_C03_P010747_S00_N19_R1: scan:18260:NIQEPTETC[Carbamidomethyl]FEEAQK/2) from 014921 in this study. (D) $\mathrm{MS}_{2}$ spectrum of "NIQEPTETCFEEAQK" from 014921 in PeptideAtlas. 


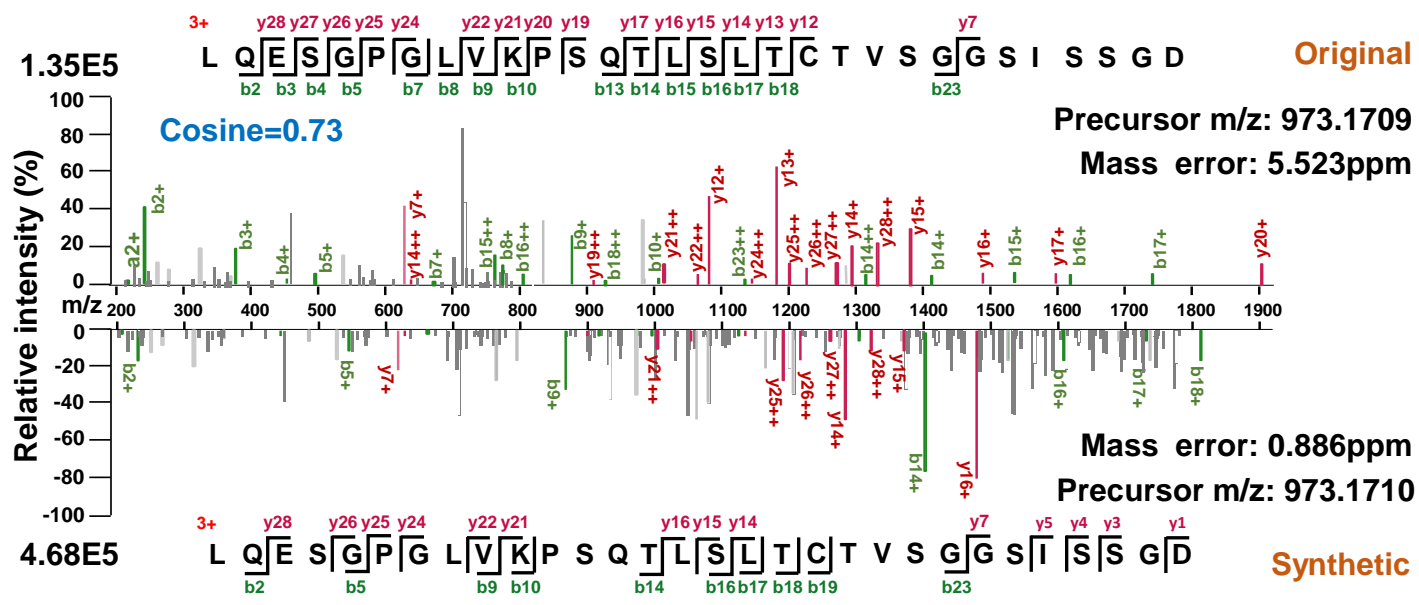

Figure S11. Verification of the identified peptide "LQESGPGLVKPSQTLSLTCTVSGGSISSGD" (mzspec:PXD021391:01294_G01_P013197_S00_N07_R1:scan:30519:LQESGPG LVKPSQTLSLTCTVSGGSISS[Ser->Val]GD/3) from P0DP06 by synthetic peptide. 
A

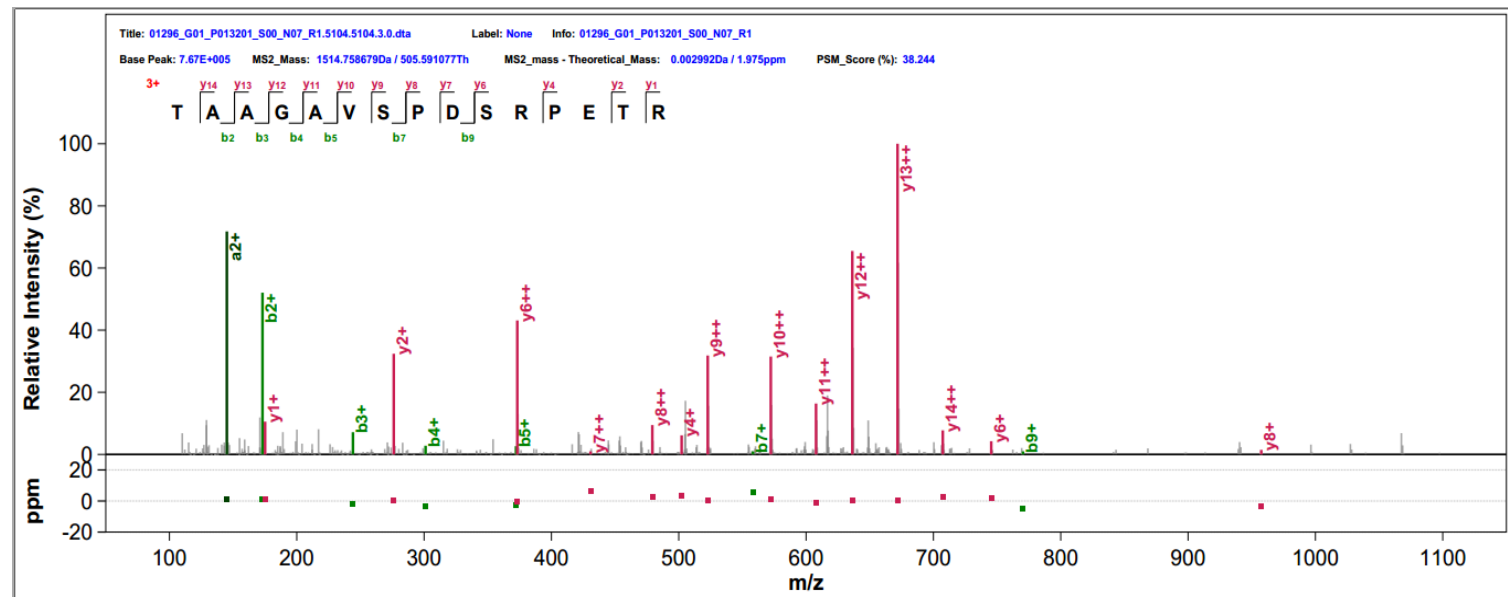

B

Spectrum for TAAGAVSPDSRPETR ${ }^{+2}$

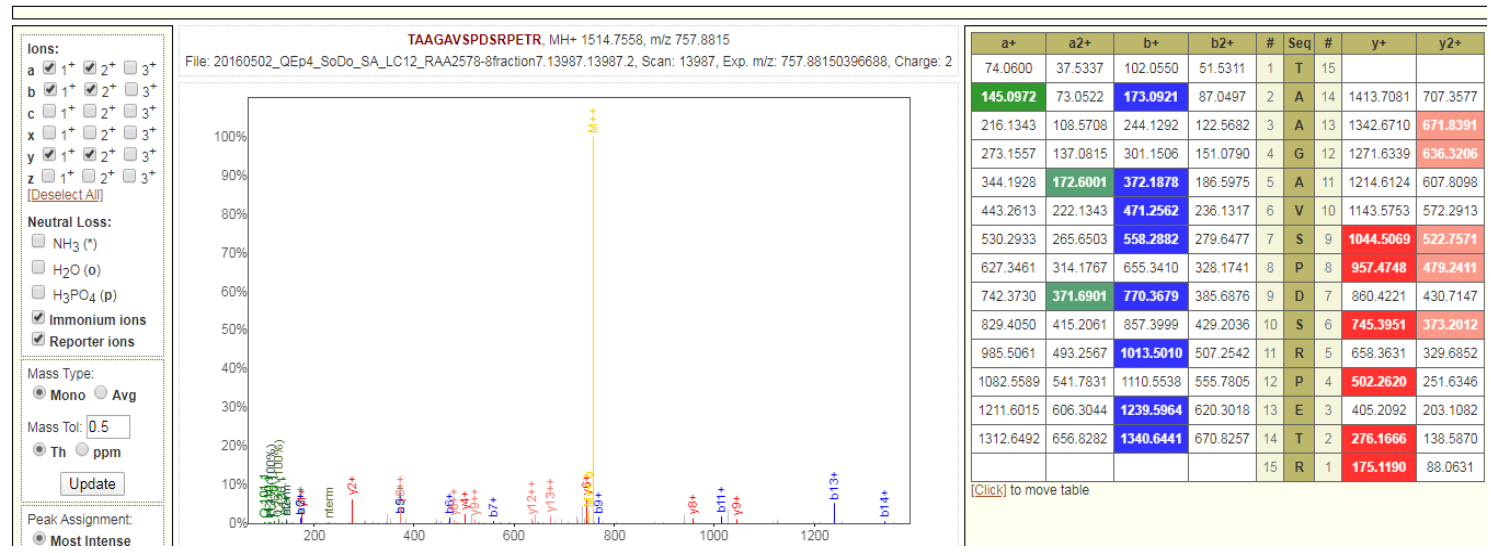


C

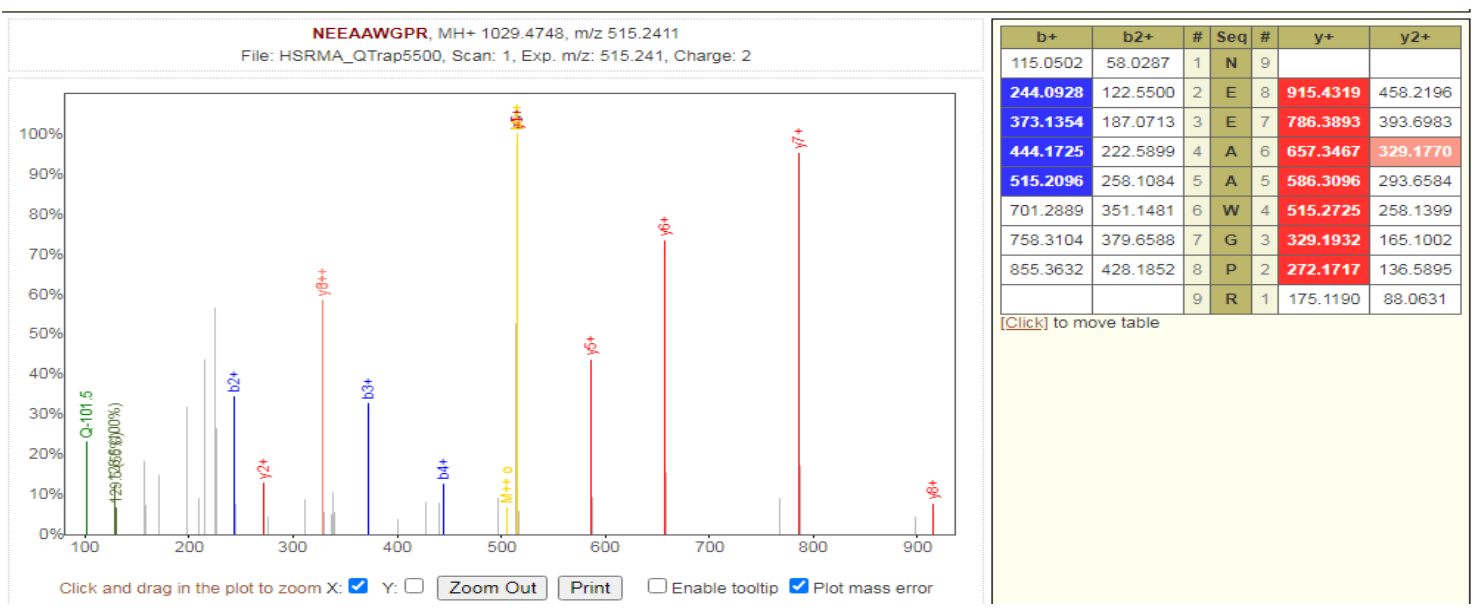

D

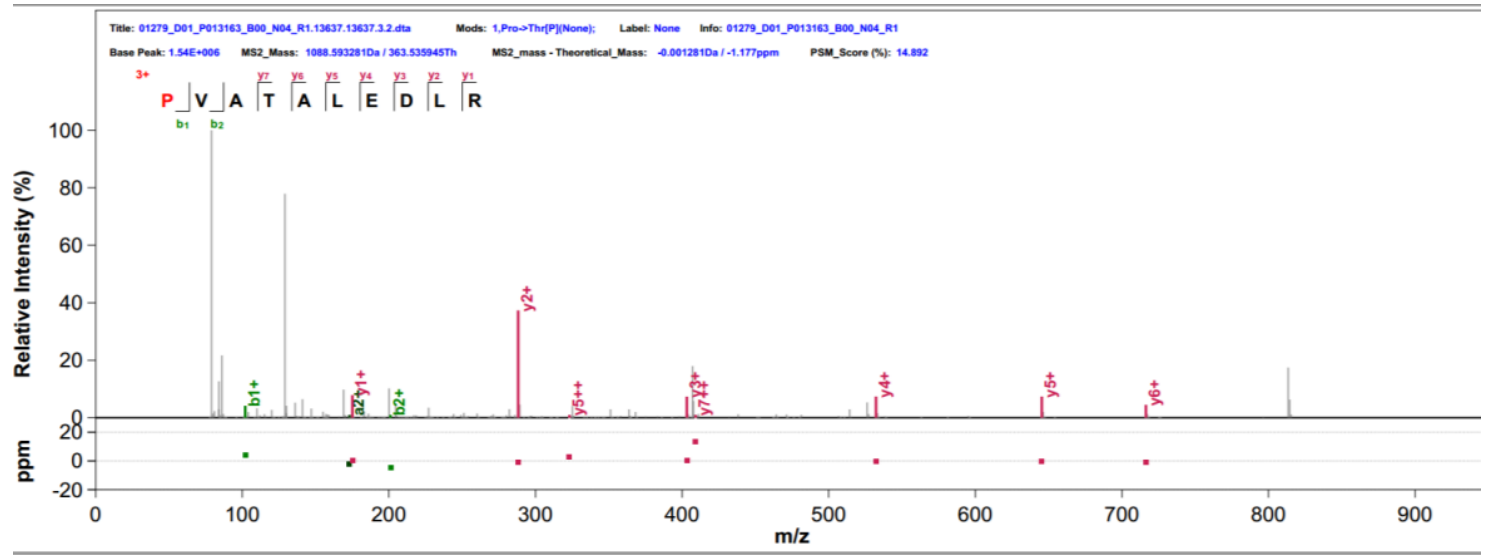

Figure S12. Verification of the identified unique peptide from Q8N912 by PeptideAtlas documented peptides. (A) $\mathrm{MS}_{2}$ spectrum of "TAAGAVSPDSRPETR"(mzspec:

PXD021391:01296_G01_P013201_S00_N07_R1:scan:5104:TAAGAVSPDSRPETR/ 3) from Q8N912 in this study. (B) MS ${ }_{2}$ spectrum of "TAAGAVSPDSRPETR" from Q8N912 in PeptideAtlas. (C) Another complement $\mathrm{MS}_{2}$ spectrum of "NEEAAWGPR" from Q8N912 in SRMAtlas. (D) $\mathrm{MS}_{2}$ spectrum of "PVATALEDLR"(mzspec:PXD021391:01279_D01_P013163_B00_N04_R1:scan:136 37:P[Pro->Thr]VATALEDLR/3) from Q8N912 in this study. 